\title{
INDEX
}

Ackerman, J.A., 144

Agerbeek, Rob, 203, 223, 225

Akihary, Monica, 23, 293, 294

Alisjahbana, Sutan Takdir, 16n14

Als je nog zingen kunt, 14, 76

Alus (concept of refinement), 88, 247

Anansi kontra Masra Bobo, 301, 308-309

Amboina Serenaders, 286

Amsterdam, 23, 75, 155, 161, 179, 199, 205, 234, 236, 252, 253, 256, 272, 290, 320, 325, $327,329,331,333$

Amsterdam Conservatory, 271, 273

Andriesen, Louis, 146, 147

Anthem

national anthem, $7,16,19,21,240$

Indonesia Raya, 25, 80-83

Wiens Neêrlands bloed, 61, 71, 79-80

Wilhelmus, 61, 73, 74, 78, 79-8o

Wilhelmus rebellions, $81-82$

Arabische Doodenzang, 155

Ardjoeno, 252

Arends, Renate, 164

Arjuna Wiwaha, 244

Astari, Bernadeta, 165

Atmadarsana, R., 129, 137-139, 146

Attima (opera), 11, 153, 155, 156, 158, 163, 166-177

Aubrey, Joyce, 286

Authenticity, 11, 15, 37, 52, 170, 176, 261, 274, 290

Baarspul, Ivan, 142

Babad Giyanti, 88

Babad Krama Dalem, 91

Babar Lajar, 231, 255

Bading, Henk, 129, 144

Bandara, Linda, 15, 129, 151, 155, 160

Bandung, 13, 18, 132, 137, 141, 142, 145, 183, $185^{-188,190,193,203,205^{-206, ~ 214, ~ 216, ~}}$ 219-220, 225, 273, 274

Banjar Gruppe, 147

Bake, Arnold, 8

Bali, 8, 9, 49, 50-52, 55-56, 58, 99, 147, 187-188, 193, 196n21, 245, 249, 252, 254

Balfoort, Dirk, 164

Batak, 68-69, 74, 115

Batavia, 11, 75, 78, 80, 90, 132, 139, 140, $142-143,145,170,176,184,197$
Bataviaasch Genootschap voor Kunsten en Wetenschappen, 267

Batu Badaun, 292, 325, 327

Bau Bau, 109, 120 court city (kraton), 115

Begdja the gamelan boy: A story from the isle ofJava (1953), 231

Belloni, Fred, 129, 144, 145, 152, 232, 254, 273

Bencoolen (also Bengkulu), 196

Berg, E.J. van der, 109, 115-120, 121, 126

Berlin, 136, 144, 161, 244, 246-247, 255, 276, 292

Betawi, 92, 95,

Beukhof, Jan, 266-268, 277

Bhaba, Homi, 110, 126 spheres of activity, 110, 113

Bintang Mas, 253

Bintang Merah, 325

Birvadda Warawidya, 138

Black Diamonds, 287

Black Magic, 287

Blokzijl, Max, 274-277

'Blown fifth' theory, 205, 207-208

Blue Diamonds, 21

Boedi Oetoemo, 132,

Boi Akih, 293

Bonnet, Rudolf, 196

Börger, F., 67, 69

Borneo, 9, 38-40, 46, 58-59, 110, 180

Bouwmeester, Frits, 234

Boven Digoel, 16, 18, 81

Brandts Buys, J.S., 9, 13, 15, 160, 186-187, 190, 194, 275

Brass bands, 92, 95, 105, 111, 253

marching bands, 89

military bands, 7ng, 19, 75, 90

Turkish military bands, 113

Broekveldt, Jan Leonard (see also

Kamadjojo, Indra), 23, 256, 257

Buka mata sama sama, 328, 333

Buton, 6, 109-111

Cabaret, 5, 253, 254, 257, 270, 297, 305, 306

California Institute of the Arts, 100

Campursari, 97, 103-105

Cartes-de-visite, 10, 46, 56

Catholicism, 7n7, 61, 75, 137

Cengkok, 130, 138, 243 
Céphas, Kassian, 34-35, 37, 46, 56, 57-59

Chen, Bubi, 203, 220, 222-223

Cheyenne, 289, 290

Chinese, 21, 35, 90, 95, 160, 237, 271, 323

Christianity, 67-68, 159, 168, 196, 209n11, $263,264,266-267,282,283,284,288,289$

Cianjuran (see also Tembang Sunda), 13, 14, 203, 211-220, 223, 226, 228, 229, 240, 243

Class, 2, 3, 5,6, 7, 11, 12, 20, 15, 93, 129, 205, 234, 235, 259, 260, 263, 264, 265, 269, 270, $273,275,313,330 n 4$

Clay, Jacob, 185

Cleber, Jos, 25, 81

Cokrowasito (also Tjokrowasito), 97, 99, 100-101, 135

Collectivism, 319, 324

Collective identity, 5, 17, 23, 80, 239, 248, 294

Colonial Institute Amsterdam, 179,199, 256

Committee for the Development of Javanese Culture, 185

Concordia Respavae Crescunt (CRC) orchestra, 145

Copyright, 273

Coolemans, Fred, 255

Counterpoint (technique), 138

Couperus, Louis, 184

Couturier, Louis, 169

Cowell, Henry, 249

Cultural polemic, 15, n14

Czechoslovakian musicians, 140

Dance

bedoyo, 34, 35

bedhaya Semang, 88, 96-97, 105, 106 bedhaya Ketawang, 96

beksan, 34

cakaiba, 49, $5^{\circ}$

galangi, 120-12, 126

gandrung Bali, 49, 50, 52, 55, 56,

gambuh, 49

Javanese dances, 37, 40, 50, 153, 163, 166, $169,170,174,175,176,231,232,240$, $245^{-247}, 248,252,254,256,305,307$, $308,311,316$

janger, 254

kuda kepang, 49, 56, 300, 311

randai, 254

sanduran Madura, 49

serimpi (also srimpi), 34, 35, 87, 88, 95,

96, 199, 245

tayub, 300

topeng, 49, 50, 52, 238, 252

war dance, 118, 121, 193n18, 283

wireng, 193
Dangdut, 104

Dangiang Parahiangan, 203, 218, 223-225

Danskunst in Indonesië (1947), 231

Dayak, 37-38, 58, 240

De Staat, 147

Diemen, Governor-General van, 112, 115, 120

Debusy, Claude, 10, 11, 143, 156, 165, 245

Demak, 144

Dengan Harapan, 319, 325-326, 336

Den Haag (also The Hague), 13, 20, 21n19, $61,134,144,148,151,153 n 8,155,156,158$, 16on11, 161,162, 163, 164, 166-170, 173, 175, 177, 178, 219n27, 223, 239, 241, 243, 245, 254, 257,26o, 274, 286n4, 328

Delft Student Union, 238

Demmeni, Jean, 39-41, 46, 56-59

Depok, 266, 267

Dewantara, Ki Hadjar (see also Soerjaningrat, R.M. Soewardi), 15 , 129-132, 135, 137, 139, 146, 242, 243

DJ Tiësto, 25

Djadjadiningrat, Hoesein, 185, 192

Djåwå, 186n8, 187, 192-193

Djojodipoero, R.T., 186

Doelwijt, Thea, 297-298, 301-304, 310, $312-313$

Doe Theatre 297

Dolle Hans, 235

Douwes Dekker, Ernest, 132

Dragonfly, 287

Dresden, Sem, 11, 165-166

Dungga, J.A., 15

Dutch Antilles, 4, 61, 83

East Timor, 322, 323, 331, 336, 337

Education, 5, 14-15, 68, 73, 75-77, 84, 129, 131, 140, 186, 187, 190, 198, 206-208, 214, 216, 242,268

vocational schools ('kweekschool'), 14, $16,129,135,137,145,219$

educational department, 77

educational policy, 79

Eerste Koloniaal Onderwijscongres, 134

Taman Siswa, 135, 242

Colonial Education Congress 1916, 243

Eighem, van, 194

Erasmus Huis, 24n22, 293 n10

Ethical Policy, 10, 13, 49, 76, 209

Ethnomusicology, 25, 136, 179n1, 205, 211, 212n19

Etnomusicologisch Centrum Jaap Kunst, 205

Eurasia, 161, 249, 251, 253, 255

Evolutionism, 31 
Exhibitions, 10, 46, 155-156, 238, 244, 253 World exhibition Paris 1889/1900, 156 Arnhem 1879, 155

Amsterdam 1833, 75, 155

Berlin 1925, 246

The Hague 1898

Exposition Coloniale Internationale, Paris 1931, 253

Exile, 81, 133, 242, 285, 319, 320-323, 330, 334

Exoticism, 2, 9, 11, 31-33, 35, 37, 40, 46, 49, 50, 56-59, 88, 153, 155, 162, 165, 171, 175-177, 238, 248, 255n19, 284

Fabricius, Jan, 232, 234-237, 275

Fairs, 9, 10, 18, 46, 49-52, 57-58, 241, 260

Ferianto, Djaduk, 4, 98-99

Film, 4, 17, 18, 19, 105, 238, 252-254, 259, 271

Fitzwilliams, O., 50

Flag

flag parade, 74

flag raising, $65,71,77,80,82$

Dutch flag, 17,69

flag reminiscent of VOC, 110, 112, 115, 120, $125^{-126}$

Flores, 196

Fock, Dirk, 151

Folk song, 6, 66, 276, 288n6, 289, 292

Fort Vredenburg, 24

Fretes, George de, 23, 285-286

Gamelan, 9, 10, 11, 15, 18, 25, 26, 33-35, 37, 46, 49, 52, 75, 87-106, 130, 135-136, 143-147, 153, 155-156, 160-161, 164-170, 173-177, 179ni, 181, 185-187, 189-190, 192, 194, 197, 199, 204-206, 209, 215, 231, 232, 238-240, 242-249, 254-256, 268, 276, 277, 300, 304-305, 307, 308, 311, 316

pelog, 11, 91-93, 253, 136, 138-139, 156, 163, 165,175, 188, 191n14, 205-206, 208-209, 211, 212, 215-216, 222-223, 229, 248, 253 slendro (also salendro), 35, 91-93, 102, $136,138,156,165,188,191 n 14,205,207$, $209 n 12$

senggani, 91

balungan, 93, 243

concept of irama, 93-95

cengkok-wilet pattern, 130,138

gendhing, 6, 130, 131, 134, 136, 138, 144, 240, 243

Australian GengGong ensemble, 101

Ensemble Gending, 26, 102

Gauthier, Eva, 25, 162,

Gelder, Max van, 274

Gendhing mares, 87-88, 91-97, 100-101
Gendhing sabrangan, 92

Genootschap van Nederlandse Componisten, 272

Gerakan Pattimura, 322

Gerharz, Nico J., 142

Geuns, van, 144

God Shiva (1955), 231

Golden Earring, 24, 289n8

Grainger, Percy, 249

Great Crash of 1929, 197

Groneman, Isaac, 9, 33-37, 58-59, 89

Grönloh, Anneke, 21

Gusti Siti, 200

H-Gang, 23, 291, 319-320, 324-337

Haarlem, 231

Haase-Peineman, Sophie, 145

Hamengkoe Boewono (also Hamengku

Buwana)

VII, 33, 92, 96

VIII, 92

$\mathrm{V}, 92$

$\mathrm{X}, 97$

Hardjodirenggo, Raden S., 252, 254

Hawaiian, 5, 13, 19, 20, 253-255, 260, 282, 284-286

Hierarchy, 19, 324

High Art (European High Art), 9, 19, 242

Hindia Poetra, 136, 241

History

historiography, 15, 26, 80, 95, 125-126

Hofland, S., 186

Hood, Mantle, 25, 146, 205n5, 208ng, 226, 231

Hullebroeck, Emiel Leopold, 129, 144, 276, 277

Hungarian musicians, 140

Huizinga, Johan, 194, 204

Hybridity, 6, 11-12, 16nı4, 20, 23, 87-92, 95, 97-106, 146, 177, 235, 274-276, 284n1, 288

Honimoa Singers, 285

Hornbostel, Erich von, 205n6, 246

Ido, Victor (also Hans van de Wall), 144, 160, 233-234, 237

IJzerdraat, Bernard, 231, 255, 256

Inayat Khan, Hazrat, 241, 248

Indische Bond, 269, 270

Indische Club, 161, 249, 253

Indisch

Indisch classical music, 151, 163, 177

Indische composers, 11-12, 151n2, 153-155, $162,163,176$

Indisch identity, 18, 20-22, 151n1, 176, 257 
Indisch fraternities, 161

Indische Kunstavond, 16, 232, 238-239, 274

Indische Kunstkring ('Art Circle'), 143, 242

Indisch opera (see also Komedie Stamboel), 151, 160, 265, 269

Indische toneel, 233

Indische Club Amsterdam, 161, 249, 253

Indische Vereeniging, 239

Indische Partij, 132

Indo

Indo Culture, 20, 24, 151n1, 160, 168, 176-177, 237, 257, 259, 265, 268, 271, 273

Indo community, 2, 83, 260, 264, 269, 275,282

Indo-Europeesch Verbond, 273, 275

Indo rock, 20-21, 285-288

Industry

entertainment / music industry, 2, 9, 11, $17,19,23,104,253,276$

Institut Seni Indonesia (ISI), 96

Akademi Seni Tari Indonesia (ASTI), 96, 220

Insulinde, 156, 161, 175

Islam, 3, 8, 19, 110-111, 125, 132, 153, 158, 159, 222n21, 269, 282, 283, 289, 307, 316

prophet Muhammad, 111

Maulid, 111

Israëls, Isaac, 184

Italian street musicians, 140

Jaap Kunst Foundation, 205n5

Jansen, Jos, 26, 218n26

Japanese

Japanese camps 18, 74

Japanese occupation, 2, 16, 17, 63, 75, 79, $84,87 \mathrm{n} 2,111 \mathrm{n} 3,18 \mathrm{o}, 219,257,284$

Jaran képang, 49, 56, 300, 311

Jazz, 11, 13, 16, 17, 19, 25, 100, 141, 163, 203, 220, 222, 223, 253, 257, 284, 289n8, 293, 294, 303, 305

Java, 3, 7n8, 12, 16, 33, 50, 81, 83, 132, 134, 143-144, 168, 169-170, 174-177, 181, 185, 205, 237, 263, 282, 290, 298-290

Javanese music, 8 , 11, 23, 35, 37, 51, 58, 87-106, 111, 130, 135-136, 145, 146, 153 , 156, 16on11, 162-164,186-188, 196, 198, 200, 209, 210-211, 213, 217n23, 232-258, $273,277,303-305,307-317$

Javanese court culture, 9, 24, 34, 88, 94, $95,129,183$

Java Institute, 186-187, 190, 192, 195, 201
Javaansch Kunstenaarstrio, $25^{2}$

Jepara, 144

Kamadjojo, Indra (see also Jan Leonard Broekveldt), 23, 256-257

Karina Adinda, 237

Kasunanan court symphony orchestra, 144

Kats, J., 186, 191, 192

Kaudern, Walter, 109, 113-118, 120-122, 126

Kayak, 24

Kilima Hawaiians, 285

Kinanthie Sandoong (composition), 129-131, 134, 136-138, 243

Kitsch, 12, 106, 148, 192

Knapp, Otto, 144

KNIL (Royal Dutch East Indies Army), $37,167,284,285 \mathrm{n} 3$

Koetjita, 254

Koenen, Tilly, 158, 161, 162

Kollektief Muziek Theater (KMT), 319, 323-325, 331, 333-337

Konservatori Karawitan Bandung (KOKAR), 206

Komedie Stamboel (stambul), 148, 159, 160, $178,257,259,265,266,268-273,278$

Koninklijke Schouwburg, 239, 240

Koperberg, Sam, 186, 190-192

Kreasi baru (new creation), 98-100

Kroncong, 5, 11, 12, 13, 19, 23, 100, 104, 144, 145, 151, 159-161, 176-177, 232, 235, 237, 240, 253-255, 257, 259-278, 284, 286

Krontjongliedjes, 272-273

Kroon-van Drunen, Fred van, 145

KUA Etnika, 99

Kuiper, Klaus, 26

Kunst, Jaap, 6, 7, 9, 12, 13, 14, 15, 25, 33, 75, $78,79,146,147,160,179-201,203-213,215$, 222, 225-227, 231, 255, 256, 283

Kunst-van Wely, Katy, 181, 188, 190, 195, 196, 198, 2001

Künstlerhaus (Berlin), 244

Kurkdjian, Onnes, 46, 42, 56, 59

Kusumadinata, R. Machjar Angga (also Koesoemadinata), 13, 14, 129, 132, 137, 139, 146, 203-225

Kusumadinata's modal theory, 13, 208, 209, 210, 211, 216-217, 225

Kutei (also Kutai), 38, 142

Ladrang Mardi Goeroe, 138

Lagu-lagu Maluku, 283, 292, 293

Land, J.P.N., 33

Lange, Daniël de, 155

Langen-Driyo, 242 
Langgam Java, 104

Langkat, 142

Last, Jef, 81

Leeuw, Ton de, 9, 102, 146, 147

Legende de Borobudur, 145

Leiden, 14, 37, 184, 218, 219, 244, 247n13, 254

Lekranty, Eddy, 290, 291

Lett, A., 67, 69

Libi Span ini na ati foe Sranan, 301, 307, 308, 311, 312, 315

Lima, Lou, 23, 286

Limburg Stirum, J.P. van (GovernorGeneral), 185

Liszt, Fransz, 155

Lydia, 21

Lo'ko Julya, 289, 290, 294

Madame Sorga, 240, 242

Madura, 49, 186n8

Mahieu, August, 265, 266, 270, 271

Majapahit, 111

Makassar, 110, 111, 112

Maluku, 110, 281-295, 320, 329

Maleische Liederen, 155

Mangoenkoesoemo, Tjipto, 132

Mangkunegoro VII, 179-201

Manik, L., 15

Manusama, Alvares Theodorus, 277

Marinus, Hector, 277

Martial arts, 34, 49, 50, 52, 237, 349, 254, 300, $305,313,316$

Massada, 282, 288, 289, 290, 294, 334

Masyarakat Seni Pertunjukan Indonesia (MSPI), 109nı

Mata Hari (also Margaretha Geertruida Zelle), 238, 241

McPhee, Colin, 146

Melaka, 262, 264

Melkweg, de, 291n9, 320, 331

Memory, 2, 3, 5, 18, 19, 63, 74, 120, 261, 262, 281, 300, 337

Mena Moeria Minstrels, 286

Mentawai, 7, 19, 61-85

Merantau (choir), 319, 320, 325, 331, 333, 334, 336, 337

Meurs, F. van, 269-270, 277

Meyer, Jo, 242

Michiels, Augustijn, 90

Migration, 2, 25, 26, 140, 142, 281, 292

Military, 63, 67, 68, 73, 142, 233, 270, 286, 312,320 military music, 6n6, 7ng, 17, 19, 88, 112, $113,114,118,122,125$ military parades, 78, 80, 90, 111

military power, 62,75

Mimicry, 24, 110, 113, 119, 305

Minangkabau, 49, 50, 58, 65, 66, 74

Minorities, 20, 277, 281, 291, 333, 342, 342,

343

Minstrelsy, 11, 21, 263, 285

Mission, 7, 63, 65, 67, 69, 73, 74, 75, 115, 192, $196,247,282$

Modern

modernity, $7,8,15,16,22,31,98,103,122$, $166,175,239,252,268,294,300$

modernism, 127-149, 155, 165, 222, 246-248, 263

Moesson (see also Tong Tong), 20, 24, 177, 223,260

Moestakim, Mariëtte, 302-305, 310, 312, 313

Mohammedaansch Gebed, 155

Moluccan, 252, 285

Moluccan community, 2, 20, 23, 24, 283, $285,294,320-322,325,327,328,330$, 333

Moluccan wards (camps), 324, 334

Moluccan Moods, 289n7, 290-292

Moluccan Schooling Collective (MSK), 322, 323

Mooi Indië (Beautiful Indies) school of painting, 18, 19, 254

Morisco, 263, 264, 266, 271, 273

Moskwa Trio, 140

Multiculturalism, 15, 23, 277, 290, 297-316

Mudato, 136, 242

Multifoon, 147, 148

Musical instruments

bamboo flute, 41, 203, 217, 218, 222, 223

bendhe, 89

brass instruments, $19,65,75,87,89,90$, 92, 93, 95, 105, 111, 253

buzzing wand (o-ore), 111

cakalele, 283

clarinet, 90, 93, 113, 140

gambang, 46

ganda drum, 111, 121

gong chimes, 9, 65, 89, 110, 111, 119, 127, $157,164,165,192,223,283,289$

guitar, 20, 41, 102, 145, 151, 160, 235, 253, 259, 260, 262, 263, 264, 267, 268, 269, $271,273,274,277,283,285,287,289$, 290, 293, 324

jew's harp, 41, 44, 65, 283

kacapi, 203, 212, 215, 218, 219, 220, 222, 223,229

kacapi suling, 203n1, 216, 22, 223

kendhang (also kendang), 89, 100, 223 
kledi (mouth organ), 39, 41, 46

piano, $7 \mathrm{n} 8,130,142,144,145,153,155,156$, $158,159,163,164,175,177,178,212,217$, 220, 222, 227, 243, 253, 271, 273, 284, 305

puwi-puwi, 89

rebab, $46,93,100,135,136,167,203$, 215 n21, 243

slompret, 89

suling, 89, 203, 212, 216, 220, 222, 223, 227 tambur, 89, 111, 116, 119, 120, 121, 122, 123, 125,126

terbangan, 300

tifa, $282,283,289$

violin, 6, 136, 140, 141, 144, 145, 159, 16o, $180,182,183,203,204,215,243,264$, 271,283

Western drum, 20, 112, 116, 125

zither, 203, 211, 212, 215, 217, 219, 220, 222, 223, 227, 229, 283

Musik kontemporer (new or experimental music), 99

Musik seni (Indonesian art music), 129, 139

Mulyadi, Mus, 23

Muntilan, 137

Mustamu, Zeth, 288, 289, 290

Na Krakti Foe Mama Sranan, 311-312

Nationalism, 3, 5, 12, 16, 17, 19, 22, 61, 76, 81, $85,132,248,297,298,322,336$

Nationalist movement, $75,80,82,83,84,131$, $242,244,248,331$

Nederlandsch Indonesisch Verbond, 247, 268

Nederlands Muziek Instituut, 151, 178

New Order (government), 15, 105

Nice, 155, 156, 159, 163, 272

Nieuwenhuis, A.W., 9, 10, 37, 38, 39, 41, 58, 59

Nijmegen, 331

NIROM, 179, 199

NIWIN commission, 17

Njai, 168, 265

Noorden, Leonie van, 245

Nostalgia, 1, 4, 5, 17, 18, 19, 21n19, 22, 257, 263, 299, 336 imperialist nostalgia, 13 tempo doeloe (also tempo dulu) 4, 176

Notation, 13, 14, 130, 131, 135, 136, 187, 206, 209-214, 229

Western staff notation, 5, 33, 134, 138, 181,272

kepatihan cipher system, 13

Noteboom, Arie, 141
Nouhuys, W.G. van, 233

Noya, Frank, 24

Notosudirdjo, Franki, 14, 243

Oberstadt, Carel, 156, 166

Oertmann, Richard, 244

Opera, 151,160

Attima (opera), 11, 155, 166, 167, 168-174, 233, 242

opera companies, 140,142

Oral traditions, 136, 209, 216, 261, 282, 294, $298,308,309$

Oriental, 11, 19, 21, 135, 151, 169, 174, 177, 289

Orientalism, 21, 135, 153, 238

Paap, Wouter, 153, 173

Pantun, 151, 153, 159, 16o, 161, 163, 174, 176, 266, 269, 275, 276

Paku Alam, 100, 129, 131, 135, 186, 192, 240

Paradiso, 290-291, 292, 333

Paramaribo, 310, 313, 314

Pasaribu, Amir, 141

Pasaribu, Ben, 98

Pasar Malam, 46, 49, 50, 52, 58, 59, 178

Pasar Malam Besar, 24, 16o, 173, 26o, 286n4

Paschal, Léon, 167

Pata Limbona, 119

Pathet (mode), also patets, 135, 191, 217

Pelasula, Mingus, 285

Pemuda 20 Mai, 319, 322, 323, 325, 331, 337

Pemuda Timbol, 312

Pentjak silat (also pencak; see also martial arts), 300, 305, 307, 313, 249, 254, 257

Peppink, 245

Philippines musicians, 75n11, 141

Phonola, 273

Photography, 9, 10, 31, 31, 34, 40, 56, 57, 115-116, 189, 190, 196, 245, 247, 249 photographic representation, 31, 33, 35, $37,39,41,59$

photo studios, 10, 31, 37, 46, 52, 56, 59, 60

Pijper, Willem, 204

Pisuisse, Jean-Louis, 274, 275, 276, 277

PKI, 323, 331

Poerbatjaraka, Raden Ngabei, 244, 245

Poetro, R.M. Soorjo, 240, 241, 242

Polyphony, 138, 293

Pop Ambon, 288

Pop daearah, 23, 288

Pop, Elisabeth (a.k.a. Raden Ayou Jodjana), 248-249

Poppy \& her Popcats, 287

Portugal, 16o, 260, 262, 267, 276

Postcolonialism, 2, 3, 8, 21, 26, 257, 261 
Postcolonial theory, 1, 7

Prick van Wely, F.P.H., 268

Prisiri Stari, 310

Protestantism, 68, 75, 263, 266

Publics, 33, 177, 210, 255, 328 consumers, 9, 32, 33, 37, 46, 49, 57, 58, 59

Queen, 162, 176

Queen's birthday, 16, 17, 81, 240

Queen Wilhelmina, 62, 69, 71, 73, 78, 80, 200

Queen (Princess) Juliana, 82, 199

Queljo, Rudy de, 287

Race, 10,11, 19, 29, 31-32, 234, 236, 237, 241, 261, 275,

Raden Mas Jodjana, 168, 169, 231, 240, 245-249, 252, 255

Raden Kodrat, 170, 245

Radio, 11, 12, 16, 17, 19, 66, 67, 97, 98, 100, 105, 16o, 179n2, 199, 220, 231

Radio of the Republic of Indonesia (RRI), 25, 100

Radio Bandung, 220

Radio Philharmonic Orchestra, 142

Rahardjo, Sapto, 97, 98, 99, 100

Raraja hadji (ceremony), 115, 211

Rarjwo Sarojo, 136-138

Ratoe Timoer, 188, 190, 195, 200

Rebirth, 312-313

Reeves, Ron, 102

Reminiscences ofJava, 175

Reich, Steve, 146, 147

Republik Maluku Selatan (RMS), 284, 285, 320

Resobowo, Basuki, 334

Revival, 20, 21n19, 237n7, 26o, 261, 273, 278, $285,287,294$

Revolution, 16, 81, 140, 266, 320, 330

Revolutionary songs, 16

Rhapsodies javanaises (van de Wall), 155, 156, 165

Rhapsodie Javanaise (Schäfer), 243

Riley, Terry, 146, 147

Robinson, Tjalie, 177, 260

Roelants-de Vogel, Kitty, 180

Roesli, Harry, 222

Romahlaiselan, 249, $25^{2}$

Ronggeng, 31, 145, 171

Royal Hawaiian Minstrels, 285, 286

Rugebregt, Maurice, 23, 293

Russian musicians, 140

Ruysch, Arie, 233

Ruyneman, Danny, 272-273
Salawat, 8

Sagimin, 305

Sahuleka, Daniel, 23, 282, 289, 290, 294

Sanoesi, 274-275

Santoso, Renadi, 26, 218n26

Saparua, 79, 293

Sarekat Islam, 132

Sastra Gendhing, 130-131

Scale, 14, 215

chromatic scale, 26,138

diatonic scale, 131, 145n8

heptatonic scale, 136

pentatonic systems, 103, 174

pelog, 11, 91, 92, 93, 136, 138, 139, 156, 163 , $165,175,188,205^{-206,208, ~ 209, ~ 210 n 16, ~}$ 211, 212, 215, 216, 222, 223, 229, 248, 253

slendro (also salendro), 36, 91, 92, 102, $138,156,165,188,205,207,208,209 n 12$, $212,215,216,217,229$

sorog, 211, 212, 215, 216, 222, 223, 229

Schäfer, Dirk, 129, 144, 155, 156, 243

Schuchardt, Hugo, 267, 277

Schrieke, Bep, 180, 181, 184, 189, 197, 198

Seelig, Paul, 129, 144, 145, 151, 155, 162, 163 , $177,191,273$

Semarang, 144, 156, 175, 233, 243, 274

Seram, 321

Serat Manuhara, 243

Serat Pakem Wirama, 92, 107

Shamanism, 66

Sheet music, 265, 271, 272, 276

Sibinga, Theo Smit, 151

Siberut, 7, 19, 61-85

Siep, Willem.F., 144, 145, 270, 271, 273, 277

Sigtenhorst, Bernard van den , 129, 144, 243

Simon, L., 270

Sjukur, Abdul, 98, 99, 101

Slamat Hindia, 253

Slave musicians, 88, 92

Sligter, Jurrien, 9, 26

Social movements, 319, 322, 325, 328, 330, 334,336

Sociëteiten, 143

Society for Ethnomusicology (SEM), 205

Soebanto, Raden Mas, 247

Soegijo, Paul Gutama, 146, 147

Soehardjo, R. (also Hardjosubroto), 129, 137, $138,139,146$

Soekamto, Raden, 247

Soekarno, 25, 80

Soemitro, Raden Mas, 239, 240

Soemodihardjo, Harto, 302, 303, 304, 312

Soeripto, Raden Mas, 247

Soerjaningrat, R.M. Soewardi (see also 
Dewantara, Ki Hadjar), 15, 129-137, 139, 146, 242, 243

Soerjowinoto, Raden Mas Moekiman, 239, 245

Soeroto, Noto Raden Mas, 144, 192, 239, 242-244, 246

Soetomo, R., 132

Solo (also Surakarta), 15, 89, 95, 96, 140, 143, 175, 179, 181, 185-201, 206-208, 252n15, 254

Songs

religious songs, $69,74,76,159,288$

children songs, 26, 78, 79, 136, 206

Souvenir, 31, 46, 52, 57

Spies, Walter, 15, 193, 196n21

Stereotypes, 4, 57, 232, 237, 255, 258, 261, 306

STOVIA (medical school), 132

STSI Bandung (formerly ASTI),

Conservatory, 214, 216, 220

Studentenvereeniging ter Bevordering van Indonesische Kunst, 254

Suanda, Endo, 109

Suara Maluku Band, 289

Suara Timur, 285

Suharto, 21, 105, 26o, 320, 323, 328, 330, 337

Sukri, Uking, 14, 203, 212-213, 217-223, 226

Sulawesi, 109, 110, 120, 121, 127, 180, 239, 340, 341

Sultan La Elangi, 112

Sunda, $5,8,13,26,49,50,90,91,93,102,138$, $145,180,203-226,256$

Suharti, Theresia, 96

Supratman, W.R., 16, 8o, 81

Surabaya, 17, 46, 49, 52, 101, 143, 144, 151, 153, $174,176,181,233,237,265,285$

Surakarta (also Solo), 15, 89, 95, 96, 140, 143, $175,179,181,185^{-201}, 206-208,252 n_{15}$, 254

Surja pop, 23

Suriname, 4, 22, 23, 61, 83, 297-316

Surinamese Javanese, 22, 23, 297-316

Syncretism, 5, 15, 25, 91, 290

\section{Tabuh-Tabuhan, 147}

Tagore, Rabindranath, 8, 239, 242

Tanjidor, 6, 87, 89-96, 105

Taman Ismael Marzuki, 21

Technology, 12, 100, 102 digital technology, 26, 100, 336

recording technology, 9, 10n12, 23, 79,

161, 180, 181, 186, 196, 204, 259, 262, 271, 273

cassette, 66-67, 104, 105, 223, 288

$\mathrm{CD}, 104,109,253$

music on cylinders, 192, 262
Tembang Sunda (see also Cianjuran), 13, 14, 203, 211-220, 223, 226, 228, 229, 240, 243

The Hague (also Den Haag), 13, 20, 21n19, $61,134,144,148,151,153 n 8,155,156,158$, 16on11, 161,162, 163, 164, 166-170, 173, 175, 177, 178, 219n27, 223, 239, 241, 243, 245, $254,257,260,274,286 \mathrm{n} 4,328$

Terschelling, $182-83$

Tideman-Weijers, Berta, 151

Tielman Brothers, 20, 286, 287

Tikar Media Budaya, 109

Tio Tek Hong, 271

Tjahja Timoer, 253

Tjang, 254

Toekomstdroom, 333

Toer, Pramoedya Ananta, 82, 86

Tong Tong (see also Moesson), 20, 24, 177, 223, 260

Toonkunst van Bali, 188, 193

Toonkunst van Java, 146, 200

Topeng masks, 49, 50, 52, 107, 238, $25^{2}$

Totok, 21, 237, 275

Transcription, 9, 14, 15, 33, 130, 243

Transmission (of knowledge), 14, 66, 204, 282, 294

Trois danses javanaises (Three Javanese dances), 144, 177

Tropische Nacht (Tropical Night), 155

Tugu, 264, 266, 269, 271

Tuning (see scale) pelog, 11, 91, 92, 93, 136, 138, 139, 156, 163, 165, 175, 188, 205-206, 208, 209, 210n16, 211, 212, 215, 216, 222, 223, 229, 248, 253 slendro (also salendro), 36, 91, 92, 102, 138, 156, 165, 188, 205, 207, 208, 209n12, 212

sorog, 211, 212, 215, 216, 222, 223, 229

Tutuarima, Eddy, 290

Uit Insulinde, 270-272

Uit Preangerdreven, 145

Valerius songs, 6,78

Vereeniging Eurasia, 161, 249, 253, 255

VOC, 5, 6, 24, 75, 109-115, 120, 121, 125, 126

Voice culture, 7

Volksspelen, 49

Wagenaar, Jan, 180

Wairata, Rudi, 286

Wajang legende (A Wayang Legend), 155

Waldjinah, 23

Wall, Constant van de, 11, 25, 129, 144, 145, 151-177 
Wall, Hans van de (a.k.a. Viktor Ido), 139, $144,156,160,233-234,237$

Waltz, 90-91

Wayang kulit, 188, 244, 252, 254, 342

Wayang wong, 34, 58, 252, 254, 256, 300

Weber, Gieneke (Siti Soendari), 245-247

Wehle, Charles, 143, 265

Weingartner, Felix, 144

Weltevreden, 170, 264

Wermeskerken, Henri van, 234

Wibisono, Joss, 25n23

Wiemans, Frans, 142, 151, 164

Wiener Schramelorkest, 141

Winternachten (festival), 24

Wirangrong, 138

Wiradiredja, Yus, 217-219

Witsen, Willem, 184

Woesthof, Dinand, 24

Wolio, 110, 119, 211, 122, 125, 126

World Music, 23, 282, 292, 293
World War II, 9, 61, 115, 180, 184, 185, 211, 232, $248,284,285$

Wullur, Sinta, 26, 163, 218

Yampolsky, Philip, 12, 95, 109

Yogyakarta, 3, 24, 33, 34, 87-92, 95-98, 100, 101, 116, 129, 135, 140, 142, 143, 151, 175, 177, 180, 186, 204, 240, 245-247, 249, 253,

254

Yogyakarta Art Festival (YGF), 97, 98, 101, 102, 105

Yogyakarta Arts Council, 97

Youth Pledge (Sumpah Pemuda), 16, 80, 252, 331

Zanten, Cornelie van, 167

Zelle, Margaretha Geertruida (also Mata Hari), 238, 240

Zwart, James, 142

Zwier, Onno, 232n7 